\title{
EFEITO DA VARIAÇÃO DO PH E DA TEMPERATURA DE SÍNTESE NO DESEMPENHO DE ADESIVOS À BASE DE CREOSOTO VEGETAL DESMETILADO-FORMALDEÍDO ${ }^{1}$
}

\author{
Crístian Bacelar Santos ${ }^{2}$, Alexandre Santos Pimenta ${ }^{3}$, Benedito Rocha Vital ${ }^{4}$ e Luiz Cláudio de A. Barbosa ${ }^{5}$
}

\begin{abstract}
RESUMO - O objetivo deste trabalho foi a produção de adesivos para madeira a partir de creosoto desmetilado. Para isto, foram sintetizadas diferentes formulações de adesivos para madeira a partir de creosoto desmetilado e paraformaldeído, variando-se o pH inicial da formulação $(12,00,12,25,12,50,12,75,13,00,13,25$ e 13,50) e a temperatura de síntese $\left(60\right.$ e $\left.70^{\circ} \mathrm{C}\right)$, para possibilitar a verificação dos melhores $\mathrm{pHs}$ e da melhor temperatura para elaboração do adesivo. Foi verificado, por meio da avaliação do gel time, que a melhor temperatura de síntese foi $70{ }^{\circ} \mathrm{C}$. Foram realizadas, então, análises por DSC (calorimetria diferencial exploratória), para obtenção dos parâmetros cinéticos e caracterização das formulações adesivas de creosoto desmetilado sintetizadas a $70^{\circ} \mathrm{C}$. Os adesivos foram utilizados para colagem, a quente $\left(160^{\circ} \mathrm{C}\right)$ e sob pressão $\left(12 \mathrm{kgf} / \mathrm{cm}^{2}\right)$, de lâminas de Araucaria angustifolia. Após a colagem, foram realizados os ensaios de resistência mecânica ao cisalhamento por tração, para condição seca e úmida. Verificou-se que a resistência da linha de cola e a porcentagem de falha na madeira para os adesivos de creosoto desmetilado sintetizados a $70^{\circ} \mathrm{C}$ foram inferiores às do adesivo de fenol-formaldeído sintetizado em laboratório, que foi utilizado como padrão.
\end{abstract}

Palavras-chave: Creosoto vegetal, desmetilação e adesivos.

\section{EFFECT PH VARIATION AND SYNTHESIS TEMPERATURE ON THE PERFORMANCE OF THERMOSETTING ADHESIVE FORMULATIONS BASED ON DEMETHYLATED WOOD CREOSOTE-FORMALDEHYDE}

\begin{abstract}
The objective of this work was the production of adhesives based on demethylated creosote for bonding of wood. Different formulations of demethylated wood bcreosote/formaldehyde were evaluated aiming at improving their bonding properties. The initial $\mathrm{pH}$ value and the synthesis temperature were varied in order to obtain the best values for producing the adhesives. Thus, seven $\mathrm{pH}$ values $(12.00,12.25,12.50,12.75,13.00$, 13.25, and 13.50) and two different temperatures $\left(60^{\circ} \mathrm{C}\right.$ and $\left.70^{\circ} \mathrm{C}\right)$ were applied. It was observed by gel time evaluation that the best synthesis temperature was $70^{\circ} \mathrm{C}$. The kinetic parameters were obtained through DSC analysis (differential scanning calorimetry) to characterize the demethylated creosote adhesive formulations synthesized at $70{ }^{\circ} \mathrm{C}$. The adhesives were used for hot bonding $\left(160^{\circ} \mathrm{C}\right)$ and under pressure $\left(12 \mathrm{kgf} / \mathrm{cm}^{2}\right)$ on Araucaria angustifolia sheets. After bonding, mechanical resistance tests were carried out under both dry and wet conditions. It was verified that shear strength and wood failure under dry and wet conditions for demethilated wood creosote-based adhesives synthesized at $70{ }^{\circ} \mathrm{C}$ were lower than for laboratory-synthesized phenolformaldehyde adhesive, used as standard.
\end{abstract}

Key words: $\quad$ Wood creosote, demethylation and adhesives.

1 Recebido para publicação em 1.8.2001.

Aceito para publicação em 9.9.2003.

2 M.S. Ciência Florestal, Dep. de Engenharia Florestal da Universidade Federal de Viçosa - UFV, 36570-000 Viçosa-MG, <bacelaros@bol.com.br>; ${ }^{3}$ Prof. Adjunto II, Dep. de Engenharia Florestal - UFV, <apimenta@ufv.br>; ${ }^{4}$ Prof. Titular, Dep. de Engenharia Florestal - UFV,<bvital@ufv.br>; ${ }^{5}$ Prof. Titular, Dep. de Química - UFV, <lcab@ufv.br>. 


\section{INTRODUÇÃO}

Praticamente todos os produtos sólidos de madeira, com exceção da madeira serrada, consomem algum tipo de adesivo na sua fabricação (Neiva \& Hellmeister, 1989). Moslemi (1974) e Skeist (1990) comentaram que entre os adesivos destacam-se os fenólicos, por apresentarem propriedades como alta resistência à umidade, durabilidade e resistência ao ataque de microrganismos, possibilitando, portanto, produtos colados mais duráveis. Contudo, um dos principais fatores que limitam seu uso é o custo, pois as matérias-primas desses adesivos são relativamente caras. Além disto, os fenóis utilizados em tais adesivos são de origem petroquímica, sendo, portanto, não-renováveis.

Segundo as observações de Pimenta (1991), uma fonte alternativa potencial de fenóis simples é o creosoto de eucalipto (destilado do alcatrão vegetal oriundo da pirólise da madeira). Porém, tais compostos possuem, quando comparados ao fenol, baixa reatividade em reações de hidroximetilação e policondensação gradual, necessárias à boa qualidade da resina (Pimenta, 1995). Egreja (1999) minimizou tal problema, desmetilando o creosoto com ácido bromídrico, para que os grupos metoxílicos $\left(\mathrm{OCH}_{3}\right)$ ligados à molécula fenólica fossem substituídos por grupos hidroxílicos $(\mathrm{OH})$, o que aumentou a reatividade dos compostos fenólicos com o formaldeído.

O objetivo deste trabalho foi verificar a melhor temperatura e os pHs para obtenção de um adesivo para madeira a partir da reação entre creosoto vegetal desmetilado e formaldeído.

\section{MATERIAL E MÉTODOS}

\subsection{Destilação do Alcatrão}

O alcatrão da Mannesmann Florestal S.A., obtido a partir da carbonização de Eucalyptus sp., foi destilado entre 180 e $300{ }^{\circ} \mathrm{C}$, em um balão bitubulado de fundo chato de 2,0 L, para extração do creosoto vegetal. A parte correspondente às águas ácidas, que é obtida em temperatura até $180^{\circ} \mathrm{C}$, foi descartada, assim como o piche residual.

\subsection{Desmetilação dos Compostos Presentes no Creosoto Vegetal, Utilizando Ácido Bromídrico (HBr)}

Para produção de creosoto desmetilado destinado à síntese dos adesivos creosoto desmetilado-formaldeído, utilizou-se a reação com ácido bromídrico descrita por Egreja (1999).

Procedeu-se da seguinte forma: em um balão de fundo chato de $500 \mathrm{~mL}$, adaptado a um sistema para destilação, foram misturados $154 \mathrm{~g}$ de creosoto e $113,3 \mathrm{~mL}$ de $\mathrm{HBr} 48 \%$. A mistura foi aquecida, sob agitação magnética, até atingir a temperatura de $130^{\circ} \mathrm{C}$, resfriando-se então o produto até a temperatura ambiente. $\mathrm{O}$ creosoto desmetilado foi acondicionado sob refrigeração a uma temperatura aproximada de $3{ }^{\circ} \mathrm{C}$, para posterior utilização.

\subsection{Análise por Calorimetria Diferencial Exploratória}

Para análise por calorimetria diferencial exploratória, foi utilizado o equipamento Shimadzu TA 50, pertencente ao Laboratório de Painéis e Energia da Madeira da UFV. As amostras, pesando em torno de $8,0 \mathrm{mg}$, foram colocadas em cápsulas de aço inoxidável e lacradas em uma prensa hidráulica, utilizando-se uma força de 2 toneladas, por 15 segundos. Essas cápsulas foram submetidas à varredura, a partir da temperatura ambiente até $250^{\circ} \mathrm{C}$. Para cada amostra de adesivo, foram utilizadas três taxas de aquecimento: 5,10 e $15^{\circ} \mathrm{C} / \mathrm{min}$. Os parâmetros cinéticos avaliados foram: energia de ativação, entalpia, temperatura de cura do adesivo e ordem de reação, obtidos por meio de um software, contendo o método de Ozawa.

\subsection{Adesivo de Fenol-Formaldeído}

$\mathrm{O}$ adesivo de fenol-formaldeído usado na colagem foi sintetizado a partir do procedimento descrito por Mori (1997). Em um balão de $500 \mathrm{~mL}$ de fundo chato, foram misturados 100,3 g de formaldeído, 48,45 g de fenol e $10,31 \mathrm{~g}$ de $\mathrm{NaOH} 50 \%(\mathrm{~m} / \mathrm{m})$. Essa mistura foi mantida sob refluxo por 17 minutos. Em seguida, abaixou-se a temperatura para $85^{\circ} \mathrm{C}$, mantida até o final do processo, e adicionou-se a segunda carga de $\mathrm{NaOH} 50 \%(4,8 \mathrm{~g})$. Após 35 minutos, foi aplicada a terceira carga de $\mathrm{NaOH}$ $50 \%$ (4,8 g). A quarta carga $\mathrm{NaOH}(4,8 \mathrm{~g})$ foi adicionada aos 60 minutos. Deixou-se a reação prosseguir por mais 1 hora e, então, o adesivo foi resfriado à temperatura ambiente, momento em que ele é considerado pronto.

\subsection{Adesivo de Creosoto Desmetilado-Formaldeído}

Foram sintetizados adesivos de creosoto desmetilado-formaldeído, adaptando-se o procedimento descrito 
por Garro-Galvez \& Riedl (1997) para a síntese de adesivo de pirogalol-paraformaldeído.

Foi utilizada a proporção molar de creosoto desmetilado/formaldeído $1: 1,8$ a sete diferentes pHs $(12,00$, $12,25,12,50,12,75,13,00,13,25$ e 13,50$)$ e duas temperaturas distintas $\left(60\right.$ e $\left.70^{\circ} \mathrm{C}\right)$. Os cálculos da relação molar do adesivo basearam-se na consideração de que esse creosoto desmetilado seria formado apenas por moléculas de pirogalol, ou seja, possuindo peso molecular igual a 154 .

Inicialmente, foram misturados em um béquer de $250 \mathrm{ml}$, sob agitação magnética, $70 \mathrm{~g}$ de creosoto desmetilado e $35 \mathrm{ml}$ de água. Em seguida adicionou-se, progressivamente, hidróxido de sódio $(\mathrm{NaOH}) 50 \% \mathrm{~m} / \mathrm{m}$ para correção do $\mathrm{pH}$. Esse procedimento foi feito em banho de gelo, para manter a temperatura a $25^{\circ} \mathrm{C}$, de modo que as leituras de $\mathrm{pH}$ não sofressem influências do aumento da temperatura durante a adição de $\mathrm{NaOH}$.

Após a correção do $\mathrm{pH}$, foram adicionados 29,75 g de paraformaldeído, sendo a solução transferida para um balão de $300 \mathrm{ml}$. A mistura foi então aquecida, sob agitação magnética, até a temperatura de 60 ou $70{ }^{\circ} \mathrm{C}$ (dependendo do tratamento a ser testado), durante 10 minutos. Após esse intervalo, o adesivo foi resfriado à temperatura ambiente, para então ser utilizado.

\subsection{Determinação do Teor de Sólidos dos Adesivos}

A determinação do teor de sólidos foi adaptada da norma ASTM-D-1518-60 (1994). Foram pesados aproximadamente $2 \mathrm{~g}$ de adesivo, que foram adicionados a uma placa de Petri, com areia, previamente seca em estufa a $105 \pm 3{ }^{\circ} \mathrm{C}$. A placa de Petri e a mistura adesivo e areia foram colocadas em estufa por 24 horas e, posteriormente, pesadas. Para cada adesivo, foram feitas duas repetições. O teor de sólidos foi calculado da seguinte forma:

$$
T s=\left(\frac{P i c-P f c}{P a}\right) \times 100
$$

em que $T_{s}=$ teor de sólidos em porcentagem; $\mathrm{Pa}=$ peso do adesivo; $P i c=$ peso inicial do conjunto: adesivo, areia e placa de Petri; e $P f_{c}=$ peso final do conjunto (após estufa).

\subsection{Tempo de Gelatinização}

Aproximadamente $1,0 \mathrm{~g}$ de adesivo foi colocado em um tubo de ensaio de $15,0 \times 2,0 \mathrm{~cm}$, que foi mergulhado em um banho de glicerina a $120^{\circ} \mathrm{C}$. A agitação dentro do tubo de ensaio foi feita manualmente, com um bastão de cobre. O tempo de gelatinização, para cada amostra de adesivo, foi contado a partir da imersão no banho de glicerina até o endurecimento parcial do adesivo, verificado quando este ofereceu maior resistência ao giro do bastão de cobre. Para cada adesivo foram realizadas oito repetições.

\subsection{Viscosidade}

A viscosidade do adesivo foi medida a $25^{\circ} \mathrm{C}$, utilizando um viscosímetro Thomas Stormer. Foram feitas três repetições, utilizando $100 \mathrm{~mL}$ de adesivo. O viscosímetro citado permite a medição da viscosidade de fluidos com até no máximo $4.000 \mathrm{cP}$. Nesse tipo de aparelho, um rotor, impulsionado por um pêndulo de massa igual a $161 \mathrm{~g}$, gira imerso no líquido do qual se deseja medir a viscosidade.

Para medir a viscosidade, considerou-se o tempo, em segundos, gasto pelo rotor para dar 100 voltas sobre seu próprio eixo. Esse tempo é diretamente proporcional à viscosidade do adesivo, ou seja, quanto maior a viscosidade, maior o tempo gasto.

Pela comparação com a curva de calibração do aparelho, feita com líquidos de viscosidade conhecida, estimou-se a viscosidade dos adesivos por meio da seguinte equação de regressão:

$$
\hat{X}=\frac{Y-8,4607}{0,1088} ; \mathrm{R}^{2}=94,5 \%
$$

em que $Y$ é o tempo, em segundos, medido no aparelho; e $\hat{X}$ é a viscosidade estimada para o adesivo em cP.

\subsection{Colagem das Lâminas de Madeira}

Lâminas de pinheiro brasileiro (Araucaria angustifolia (Bertol.) Kuntze), medindo 0,8 x 40,0 x $12,0 \mathrm{~cm}$, foram secas em estufa a $45^{\circ} \mathrm{C}$, até atingir umidade entre 8 e $9 \%$. Em seguida, essas lâminas foram cortadas nas seguintes dimensões: $0,6 \mathrm{~cm}$ de espessura, $38,0 \mathrm{~cm}$ de comprimento e $10,0 \mathrm{~cm}$ de largura.

Antes da aplicação, foram misturados ao adesivo $5 \% \mathrm{p} / \mathrm{p}$ de farinha de trigo, que foi utilizada como extensor. Foram aplicados $300 \mathrm{~g} / \mathrm{m}^{2}$ de adesivo (com extensor) em dupla face, de maneira uniforme. As lâminas foram coladas à temperatura de $160^{\circ} \mathrm{C}$, sob pressão de $12,0 \mathrm{kgf} / \mathrm{cm}^{2}$, durante 8 minutos.

R. Árvore, Viçosa-MG, v.27, n.4, p.551-559, 2003 
Foram coladas oito lâminas por tratamento, considerando-se cada lâmina uma repetição.

\subsection{Ensaios de Resistência ao Cisalhamento por Tração e de Falha na Madeira em Condição Seca e Úmida}

Após o acondicionamento das lâminas coladas, por 14 dias, sob umidade e temperatura ambientes, elas foram cortadas em corpos-de-prova, segundo a norma ASTM D 2339-70 (1994). Cada lâmina proporcionou 12 corposde-prova de 1 polegada de largura por 3 polegadas de comprimento. Para os testes de resistência e avaliação da falha na madeira em condição seca, foram utilizados dois terços dos corpos-de-prova cortados, ou seja, oito corpos-de-prova por repetição, totalizando 64 corposde-prova por tratamento.

Na avaliação de resistência da linha de cola ao cisalhamento por tração e de porcentagem de falha na madeira após umedecimento, seguiu-se o procedimento descrito por Marra (1992). Assim, foi selecionado cerca de um terço dos corpos-de-prova para cada repetição, isto é, quatro por repetição, totalizando, portanto, 32 corposde-prova por tratamento. Esses corpos-de-prova foram adicionados a uma autoclave e submersos em água a $45^{\circ} \mathrm{C}$. Por meio de um compressor, a pressão do ar dentro da autoclave foi mantida em $0,52 \mathrm{kgf} / \mathrm{cm}^{2}$. Essas condições foram mantidas durante 4 horas. Após o umedecimento, os corpos-de-prova foram transferidos para uma estufa, onde foram acondicionados a $65^{\circ} \mathrm{C}$, durante 15 horas. Depois desses procedimentos, foram executados os testes.

\section{RESULTADOS E DISCUSSÃO}

\subsection{Teor de Sólidos}

Os teores de sólidos para os adesivos de creosoto desmetilado estão no Quadro 1. Esses valores não variaram significativamente, mantendo-se em torno de 46 a $47 \%$. O adesivo de fenol-formaldeído apresentou teor de sólidos de 47,6\%.

Esses teores se mantiveram dentro de uma faixa adequada à colagem, de acordo com Pizzi (1983).

\subsection{Tempo de Gelatinização}

Por meio do teste F, constatou-se que o tempo de gelatinização para o adesivo sintetizado a $70{ }^{\circ} \mathrm{C}$ foi menor do que para o adesivo sintetizado a $60{ }^{\circ} \mathrm{C}$, independentemente da variação do $\mathrm{pH}$, conforme se observa no Quadro 2.

A Figura 1 ilustra a relação funcional existente entre o tempo de gelatinização e o pH. Observa-se que os tempos de gelatinização decrescem até um determinado valor de $\mathrm{pH}$, e a partir daí aumentam novamente. Quando são utilizadas as equações estimadas, verifica-se que os pontos de inflexão das curvas são de mínimos, os quais

Quadro 2 - Médias dos tempos de gelatinização, em segundos, para os adesivos de creosoto desmetilado, de acordo com a temperatura de síntese e o valor do $\mathrm{pH}$

Table 2 - Gel time averages, in seconds, for demethylated wood creosote-based adhesives in function of temperature and $\mathrm{pH}$ value

\begin{tabular}{|c|c|c|}
\hline \multirow{2}{*}{$\mathrm{pH}$} & \multicolumn{2}{|c|}{ Temperatura de Síntese } \\
\cline { 2 - 3 } & $70{ }^{\circ} \mathrm{C}$ & $60{ }^{\circ} \mathrm{C}$ \\
\hline 12,00 & $262 \mathrm{~A}$ & $506 \mathrm{~B}$ \\
12,25 & $237 \mathrm{~A}$ & $475 \mathrm{~B}$ \\
12,50 & $202 \mathrm{~A}$ & $395 \mathrm{~B}$ \\
12,75 & $248 \mathrm{~A}$ & $493 \mathrm{~B}$ \\
13,00 & $282 \mathrm{~A}$ & $539 \mathrm{~B}$ \\
13,25 & $319 \mathrm{~A}$ & $639 \mathrm{~B}$ \\
13,50 & $364 \mathrm{~A}$ & $649 \mathrm{~B}$ \\
\hline
\end{tabular}

Em cada pH (linha), A difere de B pelo teste $\mathrm{F}(\mathrm{p}<0,01)$.

Quadro 1 - Teor de sólidos, em porcentagem, para adesivos de creosoto desmetilado-formaldeído em função da temperatura de síntese e $\mathrm{pH}$

Table 1 -Percentage of solids for demethylated wood-creosote-based adhesives in function of temperature and $p H$ value

\begin{tabular}{|c|c|c|c|c|c|c|c|}
\hline \multirow{2}{*}{ Temperatura } & \multicolumn{7}{|c|}{$\mathrm{pH}$} \\
\hline & 12,00 & 12,25 & 12,50 & 12,75 & 13,00 & 13,25 & 13,50 \\
\hline $70{ }^{\circ} \mathrm{C}$ & 46,7 & 45,9 & 46,4 & 46,8 & 46,5 & 46,8 & 47,1 \\
\hline $60{ }^{\circ} \mathrm{C}$ & 46,1 & 46,3 & 47,4 & 45,8 & 46,8 & 47,0 & 46,9 \\
\hline
\end{tabular}

* média de duas repetições. 
correspondem aos pHs 12,41 e 12,45, para as curvas de temperatura de 60 e $70{ }^{\circ} \mathrm{C}$, respectivamente.

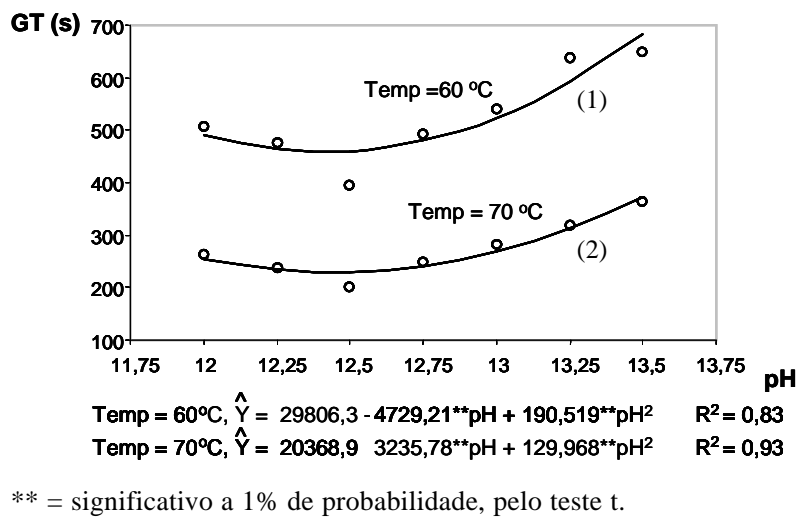

Figura 1 - Estimativa do tempo de gelatinização em função do $\mathrm{pH}$, para adesivos de creosoto desmetilado sintetizados a duas diferentes temperaturas.

Figure 1 - Gel time estimate for demethylated wood creosotebased adhesives obtained at two different temperatures and various $\mathrm{pH}$ values.

O tempo de gelatinização encontrado para a resina de fenol-formaldeído sintetizada em laboratório foi de 288 segundos. Mori (1997) encontrou, para um adesivo de fenol-formaldeído com teor de sólidos de $46 \%$, valores médios de tempo de gelatinização, a $120{ }^{\circ} \mathrm{C}$, de 265 segundos.

O tempo de gelatinização mede a reatividade do adesivo, ou seja, é um parâmetro que fornece uma noção do tempo necessário para a cura da resina (Pizzi \& Mittal, 1994).

Tomando como base os tempos de gelatinização dos adesivos de fenol-formaldeído sintetizados em laboratório e o citado por Mori (1997), decidiu-se então utilizar a temperatura de $70{ }^{\circ} \mathrm{C}$ para a síntese dos adesivos de creosoto desmetilado, pois tais resultados indicam que os adesivos sintetizados a essa temperatura se encontram em um estágio mais adequado à colagem (menores tempos de gelatinização). Após essa conclusão, passou-se a utilizar somente essa temperatura na produção de adesivos à base de creosoto desmetilado. Assim, as análises por DSC, a avaliação da viscosidade e os ensaios de resistência mecânica foram feitos para as formulações adesivas sintetizadas a essa temperatura.

\subsection{Avaliação da Viscosidade dos Adesivos}

Os valores de viscosidade encontrados após a adição do extensor (farinha de trigo), para os adesivos de creosoto desmetilado, foram superiores a $4.000 \mathrm{cP}$. O adesivo de fenol-formaldeído utilizado apresentou viscosidade de $600 \mathrm{cP}$.

Os adesivos de creosoto que possuíam viscosidade inicial entre 800 e $1.000 \mathrm{cP}$ passaram, após a adição do extensor (farinha de trigo), a apresentar viscosidade maior que $4.000 \mathrm{cP}$. A adição de extensor ao adesivo fenólico não alterou, de forma significativa, sua viscosidade.

Segundo Pizzi \& Mittal (1994), o aumento da viscosidade nos adesivos está diretamente relacionado ao aumento do peso molecular, que acontece durante sua polimerização. Portanto, seguindo o raciocínio desses autores, pode-se inferir que o crescimento da viscosidade nos adesivos de creosoto pode ter sido causado pelo aumento do tamanho de suas cadeias poliméricas, devido à ocorrência de algum tipo de ligação ou interação entre as moléculas de amido do extensor e as do adesivo à base de creosoto desmetilado-formaldeído.

\subsection{Análise por Calorimetria Diferencial Exploratória - DSC}

Para determinação das melhores formulações adesivas por meio da análise por DSC, devem ser avaliados os resultados, considerando os parâmetros cinéticos em conjunto, e não apenas isoladamente. Todavia, de modo geral, bons adesivos devem possuir menor temperatura de cura $(\mathrm{T})$, maior entalpia $(\Delta \mathrm{H})$ e menor energia de ativação (Ea). No Quadro 3, por exemplo, quando comparados dois adesivos de resinas de fenol-formaldeído, observa-se que o adesivo fenólico sintetizado em laboratório foi superior ao adesivo comercial em relação a todos os parâmetros cinéticos medidos por DSC. De acordo com Mori (1997), a superioridade do adesivo sintetizado em laboratório, neste caso, pode ser atribuída à maior pureza e à proporção mais adequada dos reagentes utilizados, o que lhe proporciona maior qualidade, segundo os parâmetros medidos.

Para a correta interpretação dos resultados de DSC, deve-se considerar que cada tipo de adesivo possui características particulares quanto à faixa de temperatura de cura, energia de ativação e entalpia. Os adesivos tânicos, por exemplo, possuem maior temperatura de cura do que os adesivos de fenol-formaldeído, o que exige maiores

R. Árvore, Viçosa-MG, v.27, n.4, p.551-559, 2003 
tempos de prensagem e temperaturas mais altas para que possam reagir, formando ligações metilênicas (Pizzi \& Mittal, 1994).

Em uma análise por DSC, a entalpia corresponde à energia absorvida pela amostra, necessária à elevação de sua temperatura, à determinada taxa de aquecimento, que é expressa em energia absorvida em relação à massa da amostra.

Considera-se energia de ativação (Ea) a quantidade de energia necessária para que as reações químicas do adesivo comecem a ocorrer. Portanto, menor energia de ativação pode indicar que o adesivo apresenta-se em bom estágio de síntese, bem como a ocorrência de uma proporção adequada de catalisador ou uma proporção maior de formaldeído, para o caso de adesivos fenólicos (Christiansen \& Gollob, 1985).
A Figura 2 ilustra os resultados de entalpia e temperatura de cura, medidas por DSC para os adesivos testados. Os adesivos de creosoto desmetilado sintetizados em diferentes pHs são comparados aos adesivos fenólicos comercial (FFC) e sintetizado em laboratório (FFL).

A temperatura de cura $(\mathrm{T})$ corresponde à temperatura necessária à cura ou plastificação do adesivo, e é determinada pelo pico da curva de aquecimento exotérmico, com taxa de aquecimento de $10{ }^{\circ} \mathrm{C} / \mathrm{min}$ (Chaney, 1994). Menor temperatura de cura implica, portanto, menores temperaturas durante a prensagem, facilitando o processo e reduzindo os custos de colagem.

Observando o Quadro 3, verifica-se que à medida que o $\mathrm{pH}$ aumenta os valores de entalpia $(\Delta \mathrm{H})$ tendem a diminuir. A mesma tendência ocorre em relação à temperatura de cura $(\mathrm{T})$.

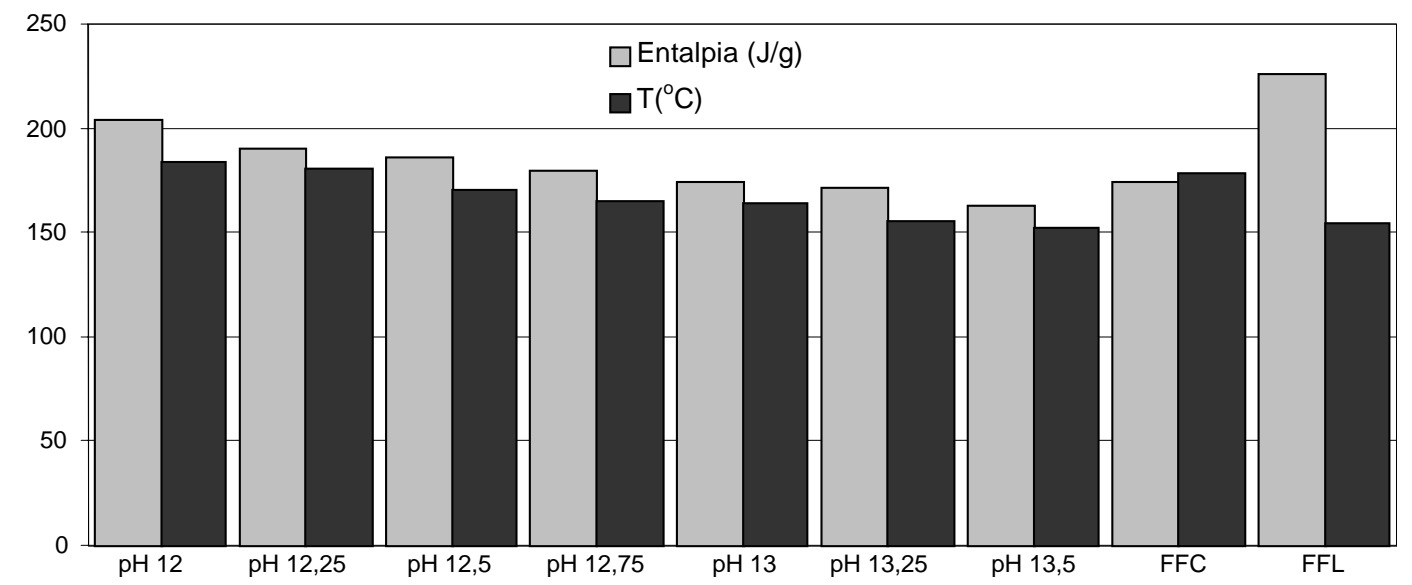

Figura 2 - Comparação da entalpia e da temperatura de cura de adesivos de creosoto desmetilado utilizados a diferentes pHs, adesivo fenólico comercial (FFC) e sintetizado em laboratório (FFL).

Figure 2 - Enthalpies and cure temperatures for demethylated wood creosotebased adhesives synthesized at different pHs, commercial phenol-formaldehyde adhesive (FFC) and phenol-formaldehyde adhesive synthesized in laboratory (FFL).

Quadro 3 - Resultados da análise de DSC para adesivos de creosoto vegetal sintetizados a $70{ }^{\circ} \mathrm{C}$ em diferentes pHs, para adesivos de fenol-formaldeído comerciais (FFC) e sintetizados em laboratório (FFL)

Table 3 - Results of the DSC analysis for wood creosote adhesives synthesized at $70{ }^{\circ} \mathrm{C}$ and different pHs, commercial phenol-formaldehyde adhesive (FFC) and phenol-formaldehyde adhesive synthesized in laboratory (FFL)

\begin{tabular}{|l|c|c|c|c|c|c|c|c|c|}
\hline \multicolumn{1}{|c|}{$\mathrm{pH}$} & 12,00 & 12,25 & 12,50 & 12,75 & 13,00 & 13,25 & 13,50 & $\mathrm{FFC}^{1 /}$ & $\mathrm{FFL}^{1 / 2}$ \\
\hline$\Delta \mathrm{H}(\mathrm{J} / \mathrm{g})^{* *}$ & 204,3 & 190,0 & 186,1 & 179,5 & 174,4 & 171,7 & 163,2 & 174,5 & 225,7 \\
\hline $\mathrm{T}\left({ }^{\mathrm{o}} \mathrm{C}\right)^{* *}$ & 184,2 & 181,1 & 170,6 & 164,9 & 163,9 & 155,9 & 151,8 & 178,3 & 154,5 \\
\hline $\mathrm{Ea}(\mathrm{KJ} / \mathrm{mol})^{*}$ & 127,3 & 120,5 & 109,7 & 114,3 & 109,0 & 118,0 & 120,6 & 93,6 & 69,14 \\
\hdashline $\mathrm{n}^{*}$ & 1,4 & 1,5 & 2,2 & 2,4 & 2,6 & 3,5 & 4,6 & 1,4 & 3,4 \\
\hline
\end{tabular}

* média referente a três taxas de aquecimento; ** referente às curvas de $10{ }^{\circ} \mathrm{C} / \mathrm{min}$.

1/ valores obtidos de Mori et al. (2001). 
A energia de ativação (Ea), entretanto, cai entre os pHs 12,00 e 13,00 (exceto para o pH 12,75) e cresce nos pHs 13,25 e 13,50.

A ordem de reação (n), que é uma classificação pelo número de moléculas que atuam sobre a velocidade da reação, também fornece uma noção do que ocorre com os adesivos quando se aumenta o pH da reação. Nota-se, então, que $\mathbf{n}$ cresce com o aumento dos valores de $\mathrm{pH}$.

Os adesivos de creosoto apresentaram bons resultados quanto à temperatura de cura, quando comparados com o adesivo fenólico comercial. A partir do pH 12,50, a temperatura de cura do adesivo de creosoto mostra-se inferior à do adesivo fenólico comercial.

A energia de ativação dos adesivos de creosoto foi superior, tanto em relação ao adesivo fenólico comercial como em relação ao de laboratório.

No caso da entalpia, o adesivo fenólico de laboratório mostrou-se sempre superior ao adesivo de creosoto. Contudo, o adesivo de creosoto apresentou valores de $\Delta \mathrm{H}$ bastante próximos daqueles do adesivo fenólico comercial.

\subsection{Ensaios de Resistência ao Cisalhamento por Tração}

Pelo teste de médias apresentado no Quadro 4, verifica-se que a resistência ao cisalhamento sob condição úmida foi menor do que sob condição seca, quando comparados os mesmos tratamentos, exceto para o adesivo sintetizado com pH 12,75, que não apresentou diferença, do ponto de vista estatístico, em nenhuma das duas condições avaliadas.

No Quadro 4 constata-se que todos os adesivos de creosoto desmetilado apresentaram menor resistência ao cisalhamento do que o adesivo de fenol-formaldeído sintetizado em laboratório, para as duas condições testadas.

A Figura 3 mostra as equações estimadas para resistência ao cisalhamento por tração sob condições secas (RCS) ou úmidas (RCU) para os adesivos de creosoto desmetilado sintetizados a $70{ }^{\circ} \mathrm{C}$.

Quadro 4 - Avaliação da resistência ao cisalhamento por tração em condições seca e úmida a diferentes $\mathrm{pHs}$

Table 4 - Shear strength results under dry and wet conditions for different $\mathrm{pHs}$

\begin{tabular}{|c|c|c|}
\hline Adesivo & $\begin{array}{c}\text { Res. em Condições } \\
\text { Secas (MPa) }\end{array}$ & $\begin{array}{c}\text { Res. em Condições } \\
\text { Úmidas (MPa) }\end{array}$ \\
\hline Fenol/formaldeído & $5,009 \mathrm{Aa}$ & $4,022 \mathrm{Ba}$ \\
Creosoto pH 12,00 & $2,487 \mathrm{Ab}$ & $1,337 \mathrm{Bb}$ \\
Creosoto pH 12,25 & $2,593 \mathrm{Ab}$ & $1,132 \mathrm{Bb}$ \\
Creosoto pH 12,50 & $2,747 \mathrm{Ab}$ & $1,110 \mathrm{Bb}$ \\
Creosoto pH 12,75 & $3,275 \mathrm{Ab}$ & $2,914 \mathrm{Ab}$ \\
Creosoto pH 13,00 & $3,669 \mathrm{Ab}$ & $2,845 \mathrm{Bb}$ \\
Creosoto pH 13,25 & $3,510 \mathrm{Ab}$ & $1,855 \mathrm{Bb}$ \\
Creosoto pH 13,50 & $2,987 \mathrm{Ab}$ & $1,987 \mathrm{Bb}$ \\
\hline
\end{tabular}

Em cada $\mathrm{pH}$ (linha), A difere de B pelo teste t pareado $(\mathrm{p}<0,05)$. Em cada resistência (coluna), a difere de b pelo teste $t$ de Student $(\mathrm{p}<0,05)$.

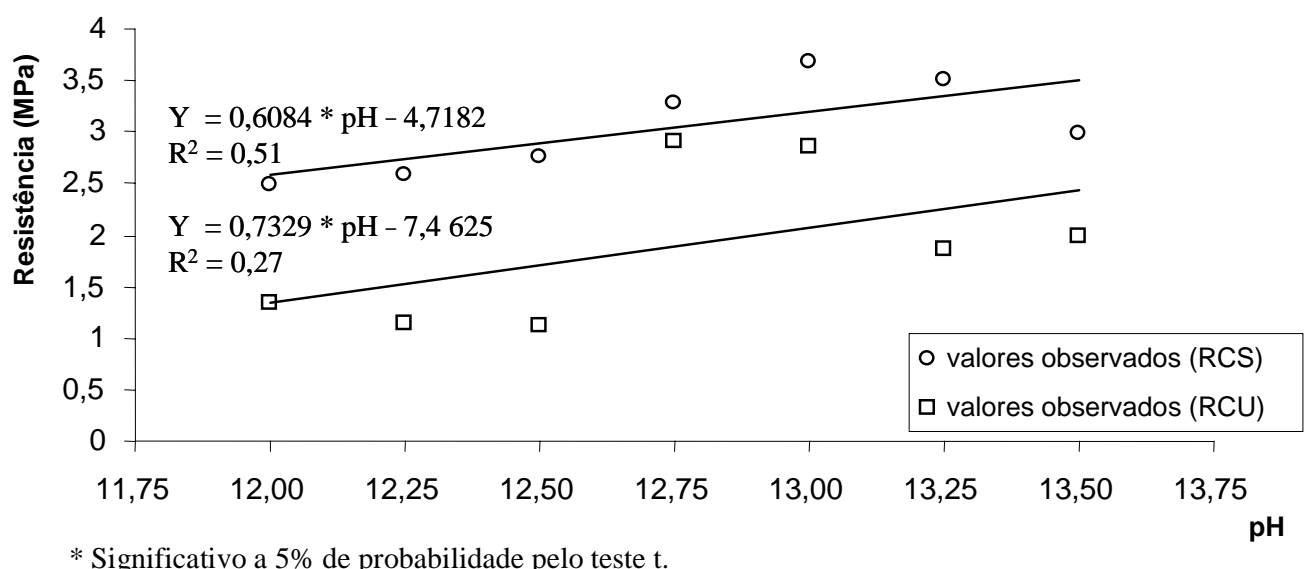

Figura 3 - Estimativa da resistência ao cisalhamento por tração, sob condições secas (RCS) e condições úmidas (RCU), dos adesivos de creosoto desmetilado sintetizados a $70{ }^{\circ} \mathrm{C}$, em diferentes valores de $\mathrm{pH}$.

Figure 3 - Estimate of shear strength under dry (RCS) and wet conditions (RCU) for demethilated wood creosote-based adhesives synthesized at $70{ }^{\circ} \mathrm{C}$ and different $\mathrm{pH}$ values. 
Quadro 5 - Teste de médias para avaliação da porcentagem de falha na madeira sob condições seca e úmida a diferentes $\mathrm{pHs}$

Table 5 - Wood failure under dry and wet conditions in different $p H s$

\begin{tabular}{|c|c|c|c|}
\hline \multicolumn{2}{|c|}{$\%$ de Falha em Condição Seca } & \multicolumn{2}{|c|}{ \% de Falha em Condição Úmida } \\
\hline Fenol/formaldeído & $94,58 \mathrm{~A} \mathrm{~A}$ & Fenol/formaldeído & $96,91 \mathrm{~A} \mathrm{a}$ \\
\hline Creosoto pH 12,75 & 49,45 A B & Creosoto pH 13,00 & $37,71 \mathrm{~A} \quad \mathrm{~b}$ \\
\hline Creosoto $\mathrm{pH} 13,50$ & $44,60 \mathrm{~A} \mathrm{bc}$ & Creosoto pH 12,75 & $35,31 \mathrm{~A} \mathrm{~b}$ \\
\hline Creosoto pH 13,00 & $43,82 \mathrm{~A} \mathrm{bc}$ & Creosoto $\mathrm{pH} 13,50$ & $17,50 \mathrm{~B}$ \\
\hline Creosoto $\mathrm{pH} 12,00$ & $33,11 \mathrm{~A}$ cd & Creosoto pH 12,50 & $13,43 \mathrm{~B}$ \\
\hline Creosoto $\mathrm{pH} 12,50$ & $24,80 \mathrm{~A}$ & Creosoto $\mathrm{pH} 13,25$ & $10,11 \mathrm{~B}$ \\
\hline Creosoto pH 12,25 & $23,14 \mathrm{~A}$ & Creosoto pH 12,00 & $8,90 \mathrm{~B}$ \\
\hline Creosoto $\mathrm{pH} 13,25$ & $22,03 \mathrm{~A}$ & Creosoto pH 12,25 & $4,04 \mathrm{~B}$ \\
\hline
\end{tabular}

Em cada pH (linha), A difere de B pelo teste t pareado $(\mathrm{p}<0,05)$. Em cada valor de falha da madeira (coluna), letras iguais correspondem a médias iguais pelo teste $t$ de Student $(\mathrm{p}<0,05)$.

Na Figura 3 nota-se que, sob condição seca, as resistências dos adesivos de creosoto aumentaram com a elevação dos valores de $\mathrm{pH}$. Sob condição úmida, apesar de os valores de resistência terem apresentado maiores variações, é possível observar a mesma tendência.

\subsection{Avaliação da Falha na Madeira}

Pode-se verificar pelo teste F, a 5\% de probabilidade, que pelo menos um tratamento difere estatisticamente dos demais quanto à porcentagem de falha na madeira para as condições úmida e seca. Apesar disto, não foi encontrado nenhum modelo de regressão adequado para avaliação das variáveis estudadas.

No Quadro 5 constata-se que todos os adesivos de creosoto desmetilado apresentaram menor porcentagem de falha na madeira em relação ao adesivo de fenol-formaldeído, para as duas condições testadas. Observa-se, também, que dentre os adesivos de creosoto desmetilado os adesivos sintetizados com pHs 12,75 e 13,50 apresentaram as maiores médias observadas para as duas condições testadas.

De acordo com Kollmann et al. (1975), Sobral Filho (1982) e Marra (1992), viscosidades muito altas prejudicam a distribuição uniforme do adesivo e a sua penetrabilidade no substrato colado, influenciando negativamente a qualidade da colagem. Assim, provavelmente as altas viscosidades dos adesivos de creosoto desmetilado-formaldeído ocasionaram os valores de resistência da linha de cola e de falha na madeira inferiores aos esperados em todos os tratamentos.

\section{CONCLUSÕES}

Após a verificação dos tempos de gelatinização das formulações adesivas à base de creosoto desmetiladoformaldeído, determinou-se que a melhor temperatura para esse tipo de adesivo, nas condições avaliadas, foi $70^{\circ} \mathrm{C}$. Após as análises por calorimetria diferencial exploratória (DSC), observou-se que, quanto à temperatura de cura e entalpia, os adesivos de creosoto desmetilado apresentaram-se comparáveis ao adesivo fenólico comercial aqui citado.

Verificou-se, entretanto, que a resistência da linha de cola e a porcentagem de falha na madeira para os adesivos de creosoto desmetilado foram inferiores às do adesivo de fenol-formaldeído sintetizado em laboratório, que foi utilizado como padrão.

Os adesivos de creosoto apresentaram alta viscosidade, não permitindo uma boa movimentação do adesivo e aderência ao substrato, o que proporciona menor qualidade à colagem. Pelo mesmo motivo, a média das resistências e a falha na madeira foram inferiores aos valores esperados.

\section{REFERÊNCIAS BIBLIOGRÁFICAS}

ASTM - AMERICAN SOCIETY FOR TESTING AND MATERIALS. Annual book of ASTM standards: Adhesives. Washington D. C.: 1994. 608 p.

BHATT, M. V.; KULKARNI, S. U. Cleavage of ethers. New York: George Tieme, 1983. p. 249-282. 
CHANEY. R. D. Characterization of phenolic and melamine thermoset systems by thermal analysis. TA Instruments, 1994.

CHRISTIANSEN, A. W.; GOLLOB. Differential scanning calorimetry of phenol-formaldehyde resols. J. Applied Polymer Science, v. 30, p. 2279-2289, 1985.

DUSWALT, A. A. The practice of obtaining kinetic data by differential scanning calorimetry. Thermochimica Acta, v. 8, p. 57-68, 1974.

EGREJA, C. M. Produção de adesivos fenólicos a partir de derivados desmetilados do alcatrão vegetal. 1999. Dissertação (Mestrado em Ciência Florestal) - Universidade Federal de Viçosa, Viçosa, 1999.

GARRO-GALVEZ, J. M.; RIEDL, B. Pyrogallolformaldehyde thermosetting adhesives. Journal of Applied Polymer Science, v. 65, p. 399-408, 1997.

LAMBUTH, A. L. Adhesives from renewable resources: Historical, perspective and wood industry needs. In: SYMPOSIUM SPONSORED BY THE CELLULOSE, PAPER, AND TEXTILE DIVISION AT THE AMERICAN CHEMICAL SOCIETY. Proceedings... New Orleans: ACS Symposium Series, 1989. p. 1-10.

KOLLMANN, F. F. P.; KUENZI, E. W.; STAMM, A. J. Principles of wood science and technology II - Wood based materials. New York: Springer-Velarg, 1975. 702 p.

MARRA, A. A. Technology of wood bonding: Principles and practice. New York: Van Nostrand Reinhold, 1992. $454 \mathrm{p}$.

MORI, F. A. Produção de adesivos para madeira com taninos da casca de Eucalyptus grandis. 1997.

Dissertação (Mestrado em Ciência Florestal) - Universidade Federal de Viçosa, Viçosa, 1997.
MORI, F. A. et al. Uso de taninos da casca de três espécies de Eucalyptus na produção de adesivos para colagem de madeira. Revista Árvore, v. 25, n. 1, p. 19-28, 2001.

MOSLEMI, A. A. Particleboard. Southern Illinois University Press, 1974. 244 p.

NEIVA, G. S.; HELLMEISTER, J. C. Estudo de resinas sintéticas em ligações estruturais em peças de madeira. In: ENCONTRO BRASILEIRO EM MADEIRAS E EM ESTRUTURAS DE MADEIRA, 3., 1989, São Carlos. Anais... São Carlos: 1989. p. 109-133.

PIMENTA, A. S. Fracionamento e análise de alcatrão e creosoto de eucalipto por cromatografia gasosa e espectrometria de massa. 1991. 68 f. Dissertação (Mestrado em Ciência Florestal) - Universidade Federal de Viçosa, Viçosa, 1991.

PIMENTA, A. S. Obtenção de adesivos fenólicos por copolimerização de fenol com alcatrão ou creosoto de eucalipto. 1995. $108 \mathrm{f}$. Tese (Doutorado em Ciência Florestal) - Universidade Federal de Viçosa, Viçosa, 1995.

PIZZI, A. Wood adhesives: chemistry and technology. New York: Marcel Dekker, 1983. 364 p.

PIZZI, A.; MITTAL, K. L. Handbook of adhesive technology. New York: Marcel Dekker, 1994. 680 p.

SKEIST, I. Handbook of adhesives. New Jersey: Chapman \& Hall, 1980. 779 p.

SOBRAL FILHO. Adesão e adesivos para madeira: Teorias da adesão e a natureza das colagens com madeira. Brasília: Instituto Brasileiro de Desenvolvimento Florestal, 1982. 22 p. (Série Técnica, 5) 